\title{
Augmentation of a Chronic Non-Healing Wound with Hydroheal Followed by Transpositional Skin Flap in a Great Dane Dog
}

\author{
M. Gokulakrishnan ${ }^{1 *}$, L. Nagarajan ${ }^{2}$, Noyingbeni Odyuo ${ }^{2}$ and K. Jothimeena ${ }^{2}$ \\ ${ }^{1}$ Department of Clinics, Madras Veterinary College, Tamil Nadu Veterinary and Animal \\ Sciences University, Chennai-600 007, India \\ ${ }^{2}$ Department of Veterinary Surgery and Radiology, Madras Veterinary College, Chenna, India \\ *Corresponding author
}

\section{A B S T R A C T}

\section{Keywords}

Chronic wound-

Hydroheal-

Transpositional

Flap - Dog

\section{Article Info}

\section{Accepted:}

05 February 2020

Available Online:

10 March 2020
An Eight-month-old intact Male Harlequin Great Dane Dog was presented to Small Animal Surgery Outpatient Unit of Madras Veterinary College with a history of a chronic non-healing wound on the lateral aspect of the distal hock region. Subjective analysis of the wound on the day of presentation revealed pink colour with purulent exudate and offensive odour. A wound planimetry was performed and the wound was dimensionally measured $(10 \mathrm{~cm} \times 12 \mathrm{~cm})$. The wound was highly exudative and was in the inflammatory phase, a wet to dry bandaging was performed on alternate days until the exudates reduced. Surgical debridement and suturing of the sulcus persistent was done in addition and placement of a drain was performed under general anaesthesia to hasten wound healing and to progress to proliferative phase. After the cessation of the inflammatory phase which was ascertained with subjective analysis the wound was treated with Hydroheal (Amorphous hydrogel with colloidal silver) on alternate days. Following good granulation, surgical intervention was planned. Since the wound contraction ceased and the presence of the wound on tension lines prevented the opposing of the recipient wound bed with standard suturing technique, an 90 degrees transposition skin flap was performed. The outcome of the surgery and uneventful recovery of the pet will be discussed further.

\section{Case history}

An Eight-month-old intact Male Harlequin Great Dane Dog was presented to Small Animal Surgery Outpatient Unit of Madras Veterinary College with a history of a chronic non-healing wound on the lateral aspect of the distal hock region. The case was previously treated by a local vet and was unresponsive to treatment. Subjective analysis of the wound on the day of presentation revealed pink colour with purulent exudate and offensive odour. A wound planimetry was performed and the wound was dimensionally measured $(10 \mathrm{~cm}$ $\mathrm{x} 12 \mathrm{~cm})$. The recurrent tarsal vein was inflamed and was not functional; hence the vein was ligated at its healthy extremities and was resected. However, due to the collateral blood supply the distal limb did not exhibit any deformities. The affected limb had 
oedema due to bandaged pressure which reduced progressively. Since the wound was highly exudative, profuse lavaging of the wound bed was done with a three-way stopper attached to a saline mixed with amikacin and an infusion set to produce a lavaging pressure of $22 \mathrm{psi}$ for effective restoration of angiogenesis and to remove the necrotic tissue. Since, the wound was in inflammatory phase a wet to dry bandaging was performed on alternate days until the exudates reduced.

Surgical debridement and suturing of the sulcus wasperformed and drain was placed under general anaesthesia to hasten wound healing and to progress the wound to proliferative phase. Subjective analysis of the wound was done on day 3, 7, 14 and 21days to assess the response to the wound treatment and to ascertain the augmentation of wound healing as opined by Plassmann, 2005.Mechanical debridement (wet - to - dry dressings) techniques was used to remove necrotic tissue remnants to avoid significant collateral tissue trauma.

More selective forms of debridement (autolytic, enzymatic) are very useful once the bulk of necrotic tissue is removed. Connery and Bellenger(2002). Selective debridement should be considered in wounds where delicate tissue structures may be otherwise damaged by surgical/mechanical debridement techniques. (Pavletic., 2003) The presence of necrotic tissue was particularly problematic since the risk of infection increased. Timely removal of dead tissue dramatically reduced the bacterial population in the wound which was assessed through a culture examination. Wet to dry dressings or mechanical debridement of residual necrotic tissue is an economical alternative to enzymatic and autolytic debridement, especially after surgical debridement has been performed to reduce the volume of necrotic tissue. (Theoret., 2005) Wet to dry dressings was considered as a form of non - selective debridement, Since both necrotic tissue and local viable tissue (epithelium, granulation tissue) were inadvertently removed during the process of removing the gauze packing. For this reason, the usage of wet todry dressings for no more than $3-5$ days is recommended.

After the cessation of the inflammatory phase which was ascertained with subjective analysis the wound was treated with Hydroheal (Amorphous hydrogel with colloidal silver) on alternate days. Establishment of a healthy vascular wound bed, free of necrotic tissue and infection, was the aim to consider all options for final wound closure.

World over researchers have shown interest in dressings impregnated with silver. Silver has antiseptic, antimicrobial, anti-inflammatory properties and is a broad-spectrum antibiotic. (Mustoe., 2004) Hydrogel silver combination provides full antimicrobial contact with entire wound bed leading to sustained and effective silver ion release. (Thomas et al., 2003)

Hydroheal consists of hydrogel component of Propylene glycerol IP $4.96 \%$ w/w and Carbomer IP $0.76 \%$ w/w, with colloidal silver in a concentration of $32 \mathrm{ppm}$. Giovanni et al., (2004) Clear antimicrobial wound gel containing colloidal silver provided full antimicrobial contact with entire wound bed and led to sustained and effective silver ion release for 3 days. Lansdown and Williams (2004) Gel dressing remained clear while in use and there was no discoloration or staining of tissue. Following good granulation, surgical intervention was planned. Since the wound contraction ceased and the presence of the wound on tension lines prevented the opposing of the recipient wound bed with standard suturing technique, an 90 degrees transposition skin flap was performed. 
Int.J.Curr.Microbiol.App.Sci (2020) 9(3): 937-941

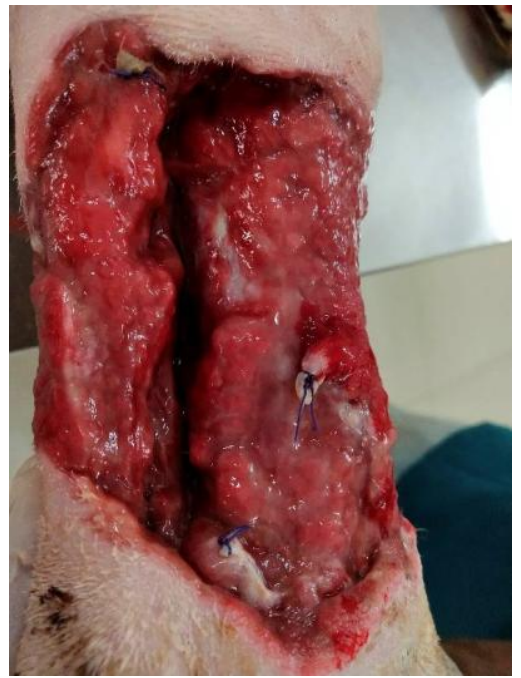

Fig.1 Chronic non-healing wound with severed recurrent tarsal vein

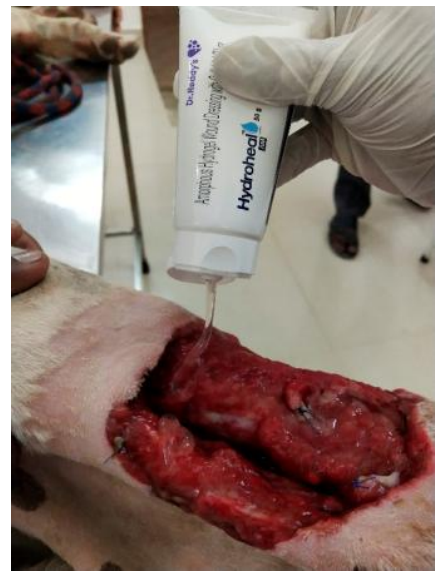

Fig.2 Application of hydroheal for wound

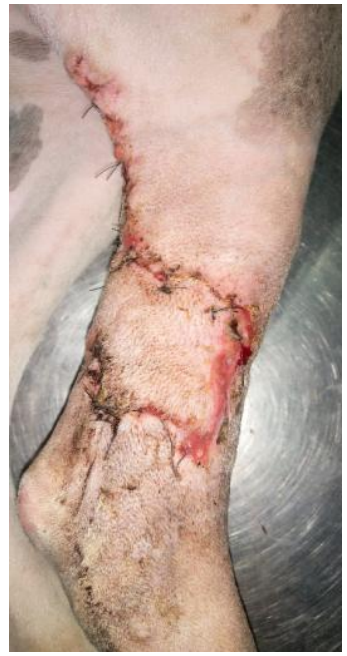

Fig.3 Transpositional skin flap with complete flap uptake 
The donor area was thoroughly clipped. Cleansing the skin with isopropyl alcohol impregnated gauze sponges helped to remove natural oils that impaired use of felt tipped marking pens. Alcohol resistant marking pens were used since the flap outline was less likely to be rubbed off during the surgical preparation of the skin. The transposition flap is a rotating flap by design and versatile of the local flaps for wound closure. Transposition flap brought additional skin to the defect.

All wounds are the result of the absorption of energy transferred to the body, whether it is from a projectile, electrical current, or a surgeon's scalpel blade.(Selwyn, 1981). The severity of the insult depends upon the strength of the energy source, how it is dispersed to the body, and the specific tissue(s) absorbing it. Mustoe et al., (2006) Understanding the processes of wound repair is essential to a thoughtful approach to wound care. Significant advances in cellular and molecular biology over the past several years have improved our understanding of the processes of wound healing and tissue regeneration. Augustin et al., (2003).If minimal necrotic tissue remains, products promoting autolytic debridement can be substituted for wet to dry dressings.(Mayrovitz and Soontupe, 2009).

Surgical Reconstruction after removal of large volumes of tissue (e.g. for treatment of neoplasia, trauma, or infection) presents special challenges to the surgeon. (Schultz et al., 2005). Rapid uncomplicated wound healing, always a primary goal of surgery, is particularly desirable for large wounds or wounds over vital structures because failure of wound repair is potentially catastrophic.

A pedicle graft or skin flap is a partially detached segment of skin and subcutaneous tissue: the base or pedicle of the flap maintains circulation to the skin during its elevation and transfer to a recipient location. (Hedlund., 2006) Pedicle graft developed adjacent to the recipient bed is termed local flaps. They represent one of the most practical methods of closing defects that cannot be approximated by simple undermining and suturing.

The effective use of these flaps usually depended on forming a flap in a neighbouring area in which loose, elastic skin prevails as performed in the present case.The 90 - degree transposition flap was particularly useful in this context. Any secondary defect created by the transfer of the flap to the defect can be closed directly. The transposition $\mathrm{fl}$ ap is adaptable to smaller defects of the limbs by developing the fl ap parallel to the length of the limb in order to take advantage of the limited circumferential skin laxity present in the dog and cat On occasion, the surgeon may note a variable amount of skin elasticity along the distant border of the cutaneous defect.

Serial (scalpel, scissors) or mechanical debridement (wet - to - dry dressings) techniques are used to remove necrotic tissue remnants, provided that significant collateral tissue trauma can be avoided.(Houghton and Campbell.,1999) More selective forms of debridement (autolytic, enzymatic) are very useful once the bulk of necrotic tissue is removed.

Establishment of a healthy vascular wound bed, free of necrotic tissue and infection, allows the surgeon to consider all options for final wound closure.(Mayhew and Holt., 2003)The intrinsic blood supply of a flap is the most critical determinant of successful transfer and is therefore the most clinically valid method of classification.(Reetz et al., 2006) Intradermal skin sutures can provide supplemental support to skin closures and significantly reduce the risk of wound dehiscence after external skin sutures are removed. 


\section{References}

Augustin, M.; Maier, K. Psychosomatic Aspects of Chronic Wounds. Dermatology and Psychosomatics 2003;4: 5.

Connery N.A. and Bellenger C.R. 2002. Surgical management of haemangiopericytoma involving the biceps femoris muscle in four dogs. Journal of Small Animal Practice. 43, 497-500. 3.

Crovetti, Giovanni; Martinelli, Giovanna; Issi, Marwan; Barone, Marilde; Guizzardi, Marco; Campanati, Barbara; Moroni, Marco; Carabelli, Angelo. Platelet gel for healing cutaneous chronic wounds. Transfusion and Apheresis Science 2004;30 (2): 145-51.

Hedlund C.S. 2006. Large Trunk wounds. Vet.Cli.N. Am. Small. Anim. Pract. 36: 847-872.

Houghton P. E. and Campbell K. E. 1999. Choosing an adjunctive therapy for the treatment of chronic wounds. Ostomy Wound Management. 45: 43-52.

Hunt G.B. 1995. Skin fold advancement for closing large sternal and inguinal wounds in cats and dogs. Vet. Surg. 24: 172.

Lansdown andWilliams. How safe is silver in wound care? J Wound Care 2004; 13(4): 131-36.

Mayhew P. D. and Holt D. E. 2003. Simultaneous use of bilateral caudal superficial epigastric axial pattern flaps for wound closure in a dog. J Small Anim Pract.44: 534-538.

Mayrovitz H.N. and Soontupe L.B. 2009. Wound areas by computerized planimetry of digital images: accuracy and reliability. Adv. Skin. Wound. Care. 22 (5): 222-9. 14.

Mustoe TA, O'Shaughnessy K, Kloeters O.
Chronic wound pathogenesis and current treatment strategies: a unifying hypothesis. J PlastReconstr Surg. 2006; 117:35-41.

Mustoe, Understanding chronic wounds: A unifying hypothesis on their pathogenesis and implications for therapy. American Journal of Surgery 2004; 187 (5): S65.

Pavletic M.M. 2003. Skin and Adnexa. Textbook of small animal surgery Ed D. Slatter, Vol.1, 3rd ed, pp 250259.Saunders.philadelphia.

Pavletic M.M. 2010. Atlas of Small Animal Wound Management and Reconstructive Surgery. Oxford: Wiley-Blackwell.

Plassmann P. 2005. Measuring wounds. J. Wound. Care. 4(6): 269-72.

Reetz J.A, Seiler G, Mayhew P.D. and Holt D.E. 2006. Ultrasonographic and color - flow Doppler ultrasonographic assessment of direct cutaneous arteries used for axial pattern skin $\mathrm{fl}$ aps in dogs. J Am Vet Med Assoc228: 1361 - 1366.

Schultz G, Mozingo D, Romanelli, M. and Claxton, K. 2005. Wound healing and TIME: new concepts and scientific applications. Wound Repair Regen13 (4 Suppl): S1-S11.

Selwyn S. Microbial interactions and antibiosis. In: Maibach H, Aly R, eds. Skin microbiology: relevance to clinical infection. New York:Springer-Verlag, 1981:63-74.

Theoret C.L. 2005. The Pathophysiology of Wound Repair. Vet Clin Equine Pract. 21:1-13.

Thomas and McCubbin P. An in vitro analysis of the antimicrobial properties of 10 silvercontaining dressings. J Wound Care 2003; $\quad$ 12(8): $305-08$.

\section{How to cite this article:}

Gokulakrishnan. M, L. Nagarajan, Noyingbeni Odyuo and Jothimeena. K. 2020. Augmentation of a Chronic Non-Healing Wound with Hydroheal Followed by Transpositional Skin Flap in a Great Dane Dog. Int.J.Curr.Microbiol.App.Sci. 9(03): 937-941.

doi: https://doi.org/10.20546/ijcmas.2020.903.110 